\title{
Determination of Symptoms of Depressive, Anxiety and Somatic and Perceived Threat of Covid-19 Among Health Care Workers
}

\author{
Sağlık Çalışanlarında Depresif, Anksiyete ve Somatik Belirtileri \\ ile Algılanan Covid-19 Tehdidinin Belirlenmesi
}

\author{
Elif Dönmez ${ }^{1}$, İknur Dolu², Hakan Karaş ${ }^{3}$
}

\begin{abstract}
:
This study aimed to determine symptoms of depressive, anxiety and somatic and perceived threat of coronavirus disease among health care workers. The study was conducted in Istanbul. 315 health care workers who completed the online questionnaire included in the study. Measurement tools consisted of a questionnaire including items to define perceived level of coronavirus disease of participants and questions about demographic characteristics and Patient Health Questionnare-9, Generalized Anxiety Disorder-7, and Patient Health Questionnaire-15. It was determined that somatic symptoms were clinically significantly high in $37.8 \%$ of the healthcare workers and it is followed by depression symptoms in $34 \%$ and anxiety symptoms in $23.5 \%$ respectively. The results showed that $77.8 \%$ of participants felt coronavirus disease as a life-threatening situation and $95.6 \%$ of them worried about being infected. Although worrying about being infected by coronavirus affected symptoms of depressive, anxiety and somatic, feeling life-threatening of coronavirus disease affected symptoms of anxiety and somatic. The factors associated with depression were following; females ( $\mathrm{OR}, 3.85, \mathrm{p}=0.001)$, being married $(\mathrm{OR}, 2.35, \mathrm{P}=0.049)$, and no physical exercise $(\mathrm{OR}, 3.02, \mathrm{p}=0.008)$. As to somatic symptoms, being female $(\mathrm{OR}, 2.63, \mathrm{p}=0.013)$, living alone $(\mathrm{OR}, 2.66$, $\mathrm{p}=0.016)$, having chronic diseases $(\mathrm{OR}, 4.31, \mathrm{p}=0.000)$, and no physical exercise $(\mathrm{OR}, 2.33, \mathrm{p}=0.025)$ were found as the risk factors. Early psychosocial and psychotherapeutic interventions to these groups that have risk factors in terms of psychiatric symptoms might be beneficial in preventing the development of psychiatric symptoms in healthcare workers during outbreak.
\end{abstract}

Keywords: Depression, anxiety, somatization, perceived threat

${ }^{1} \mathrm{PhD}$., MScN., RN., Assistant Professor, University of Health Sciences, Nursing Faculty, Oncology Nursing Department, Istanbul-Turkey, Orcid İd: https://orcid.org/0000-0001-5030-3411

${ }^{2} \mathrm{PhD}$., MScN., RN., Assistant Professor, Bartin University, Faculty of Health Science, Bartın-Turkey, Orcid İd: https://orcid.org/0000-00020958-8395

${ }^{3}$ PhD., MD., Assistant Professor, Istanbul Gelisim University, Department of Psychology, Istanbul-Turkey, Orcid İd: http://orcid.org/00000002-9391-5415

Address of Correspondence/Yazışma Adresi: Bartin University, Faculty of Health Science, Bartın-Turkey, E-mail: idolu@ bartin.edu.tr Date of Received/Geliş Tarihi: 22.04.2021, Date of Revision/Düzeltme Tarihi: 08.06.2021, Date of Acceptance/Kabul Tarihi: 14.07.2021, Date of Online Publication/Çevirimiçi Yayın Tarihi: 01.09.2021

Citing/Referans Gösterimi: Dönmez, E., Dolu, İ. \& Karaş, H. (2021). Determination of Symptoms of Depressive, Anxiety and Somatic and Perceived Threat of COVID-19 Among Health Care Workers, Cyprus Turkish Journal of Psychiatry \& Psychology, 3(3): 165-74.

(C) 2021 The Author(s). Published by Cyprus Mental Health Institute / Cyprus Turkish Journal of Psychiatry and Psychology (www.ktppdergisi.com). This article is an open access article distributed under the terms and conditions of the Creative Commons Attribution 4.0 license which permits use, sharing, adaptation, distribution and reproduction in any medium or format, provided the original work is properly cited and is not used for commercial purposes. http://creativecommons.org/licenses/by/4.0/ 


\section{Öz:}

Bu çalışmanın amacı, sağlık çalışanlarında depresif, anksiyete ve somatik belirtiler ile algılanan koronavirüs tehdidini araştırmaktır. Çalışma İstanbul'da yapılmıştır. Çalışmaya çevrimiçi anketi dolduran 315 sağlık çalışanı dahil edilmiştir. Çalışmada ölçme aracı olarak katılımcıların algılanan koronavirüs hastalığı tehdit düzeyini tanımlayan maddeler ve demografik özellikleri içern anket formu ile Hasta Sağlık Anketi-9, Genelleştirilmiş Anksiyete Bozukluğu-7 ve Hasta Sağlığı Anketi-15 kullanılmıştır. Sağlık çalışanlarının\% 37,8'inde somatik belirtilerin klinik olarak anlamlı derecede yüksek olduğu ve bunu sırasıyla\% 34'inda depresyon ve \% 23,5'inde anksiyete belirtilerinin izlediği tespit edilmiştir. Bu çalışma sonucuna göre, katılımcıların\% 77,8'inin koronavirüs hastalığını yaşamı tehdit eden bir durum olarak hissettiğini ve \% 95,6's1 enfekte olmaktan endişe duyduğunu saptanmıştır. Koravirüse yakalanma endişesi depresif, anksiyete ve somatik semptomları etkilese de, koronavirüs hastalığının yaşamı tehdit etmesi hissi anksiyete ve somatik semptomları etkilemektedir. Depresyonla ilişkili faktörler kadın olma $(\mathrm{OR}, 3,85, \mathrm{p}=0.001)$, evli olma $(\mathrm{OR}, 2,35, \mathrm{P}=0.049)$ ve fiziksel egzersiz yapma $(\mathrm{OR}, 3.02$, $\mathrm{p}=0.008)$ olarak tanımlanmıştır. Somatik belirtiler için de kadın olmak (OR, 2,63, p =0,013), yalnız yaşamak $(\mathrm{OR}, 2,66, \mathrm{p}=0,016)$, kronik hastalı̆ı olmak $(\mathrm{OR}, 4,31, \mathrm{p}=0,000)$ ve fiziksel egzersiz yapmamak $(\mathrm{OR}, 2,33, \mathrm{p}=$ $0,025)$ risk faktörü olarak bulunmuştur. Psikiyatrik belirtiler açısından risk faktörleri olan bu gruplara erken dönem psikososyal ve psikoterapötik müdahaleler, salgın sırasında sağlık çalışanlarında psikiyatrik belirti gelişimini önlemede faydalı olabileceği düşünülmektedir.

Anahtar Kelimeler: Depresyon, anksiyete, bedenselleştirme, tehdit algısı

\section{Introduction}

The coronavirus disease (COVID-19) caused by SARS$\mathrm{CoV}-2$ is a new coronavirus which was identified in Wuhan City of China in December 2019 for the first time. Fast outbreak of COVID-19 was declared as an emergency public health situation that should be concerned about by the World Health Organization (WHO). According to WHO data, it is known that 10.533 .779 people have died since the beginning of the outbreak (WHO, 2020a, 2nd July 2020). In Turkey, COVID-19 was seen for the first time on 11 March 2020. According to the data disclosed by Turkish Ministry of Health (MoH) on 25 May 2020, 157.814 people have been diagnosed with coronavirus disease and 4369 people have died because of coronavirus since the first case (MoH, 2020).

Since COVID-19 is a pandemic which spreads faster when compared to SARS, it creates more fear, panic and distress among the society. Although healthcare workers save lives of many people during COVID-19, they are exposed to more workload and infection risk. In a study conducted during early periods of COVID-19 pandemic, it is reported that $29 \%$ of the COVID patients in hospitals were healthcare workers (Zhu et al., 2020). In a statement by the International Council of Nurses (ICN), it is reported that 90.000 healthcare workers are infected with COVID-19 and approximately 260 nurses might have died due to the disease (ICN, 2020). According to a statement by CDC on 15 April, it is stated that 9200 healthcare workers are infected with COVID-19. In this period, healthcare workers have worked on the front row in fighting the pandemic and they have been exposed to dangers that put them at infection risk. Some of these dangers are being exposed to pathogen, long working hours, psychological distress, fatigue, occupational burnout, stigmatization, physical and psychological violence (WHO, 2020b).
In this period, healthcare workers who were quarantined with COVID-19 diagnosis faced social isolation and healthcare workers who were not quarantined were exposed to social discrimination (Zhu et al., 2020). For all these reasons, healthcare workers who directly take care of diagnosis, treatment and care of the COVID-19 patients experience psychological distress and their mental health is exposed to the risk of being deteriorated (Lai et al., 2020). Continuously increasing number of cases, excessive workload, running out of protective equipment, media's impact, inadequate medication and support also contribute in deterioration of healthcare workers' mental health (Lai et al., 2020). For those reasons, healthcare workers may show emotional reactions and experience psychological distress. All those reasons might cause attention deficit, difficulty in making clinical decisions and deterioration in cognitive functions among healthcare workers. Psychological state of healthcare workers is a significant public health problem because of those reasons (Zhu et al., 2020). It was reported that the most common psychological problems observed among healthcare workers who worked during COVID-19 pandemic are anxiety (Hacimusalar et al., 2020, Lai et al., 2020; Sahin et al., 2020; Tan et al., 2020), hopeless (Hacimusalar et al., 2020), stress (Tan et al., 2020), insomnia (Lai et al., 2020), distress (Lai et al., 2020), depression (Lai et al., 2020; Liang et al., 2020; Sahin et al., 2020; Tan et al., 2020), posttraumatic stress disorder symptoms ( Sahin et al., 2020; Tan et al., 2020) and emotional stress (Cai et al., 2020).

In the literature, little is known about factors related with healthcare workers' depressive, anxiety and especially somatic symptoms in Turkey (Hacimusalar Y,2020; Sahin, 2020). The aim of this study is to identify the depression, anxiety, and somatic symptoms and perceived threat of coronavirus disease among healthcare workers practicing in all level of healthcare services during COVID-19 outbreak. Another aim of the study was to investigate factors related to such psychiatric symptoms. 


\section{Method}

\section{Design and participants}

This cross-sectional survey conducted between April 18th and May 25th, 2020, when was one month after the first coronavirus case was officially confirmed by Ministry of Health of Turkey. The participants were defined as healthcare workers (HCWs) practicing at hospitals. Snowball sampling was performed to be recruited the HCWs. The healthcare workers consisted of nurses, physicians, and other healthcare workers such as paramedics, physical therapists, psychologist. The study was a multi-centered in a metropolis, Istanbul, in the west of Turkey. The online questionnaire was shared in the social media platforms of healthcare workers which can only be subscribed by phone number (WhatsApp) to deliver the participants. $315 \mathrm{HCWs}$ who completed the online questionnaire included in the study. The ethical approval of this study was obtained from the ethic committee of the University where the second author worked (decree code: 2020/54). The informed consent was the first part of the anonymous online questionnaire.

\section{Instruments}

An anonymous online questionnaire was used to collect data from healthcare workers. The measurement tools consisted of three parts: (i) informed consent, (ii) sociodemographic characteristics of healthcare workers, (iii) participants' perceived threat of COVID-19, and The Patient Health Questionnaire: Somatic Anxiety Depressive Symptoms (PHQ-SADS). The second and the third parts of the questionnaire were developed by preliminary literature review (Ho et al., 2020; Li et al., 2020; Zhu et al., 2020). Physical exercise was measured according to meeting criteria of physical activity guideline for adults published by Ministry of Health of Turkey (2018) (MoH, 2018).

The Patient Health Questionnaire: Somatic Anxiety Depressive Symptoms (PHQ-SADS): The PHQ-SADS was developed by Kroenke et al (2010) and validated by Güleç et al. (2012) in the Turkish context. The scales were developed to measure the somatic, depressive, and anxiety symptoms of patients in the primary healthcare settings. The PHQ-SADS, which have good psychometric properties, consisted of four following modules: PHQ-15 (somatoform symptoms), GAD-7 (anxiety symptoms), PHQ-9 (depressive symptoms), and 5-item panic modules. In the present study, we used only the first three modules and considered the scores of $\geq 10$ to specify the presence anxiety, depression, and somatization which is the most commonly recommended cutpoint for "clinically significant" symptoms on all three scales (Kroenke et al., 2010).

Patient Health Questionnare-9 (PHQ-9): The PHQ-9 is a short self-report scale which is used for reviewing depression symptoms and evaluate its severity. It has demonstrated high sensitivity (.88) and specificity (.88) in primary clinic populations. The total PHQ-9 score for the nine items ranges from 0 to 27 and scores of 5, 10, 15, and 20 represent cut points for mild, moderate, moderately severe, and severe depression respectively. Cronbach's alpha coefficients were found as 0.89 for the original scale and 0.88 for Turkish translation (Güleç et al., 2012, Kroenke et al., 2001).

Generalized anxiety disorder-7 (GAD-7): The GAD-7 is a self-report scale which is used for reviewing the symptoms of generalized anxiety disorder and evaluate its severity. It has been extensively used and validated in primary care settings and has been shown to have good sensitivity (.89) and specificity (.82) as a screener for generalized anxiety disorder. The total GAD-7 score for the seven items ranges from 0 to 21 ; scores of 5,10 , and 15 re-present cut points for mild, moderate and sever anxiety, respectively. Cronbach's alpha coefficients were found as 0.92 for the original scale and 0.92 for Turkish translation (Güleç et al., 2012, Spitzer et al., 2006).

Patient Health Questionnaire-15 (PHQ- 15): The Patient Health Questionnaire (PHQ-15) is a 15-item scale which is used for reviewing somatic symptoms. It has been extensively used and validated in primary care settings and has been shown to have good sensitivity (.78) and specificity (.71) as a screener for somatoform disorders (van Ravesteijn et al., 2009). The PHQ-15 scores of 5,10 , and 15 represent cut points for low, medium and high somatic symptoms severity respectively. Cronbach's alpha coefficients were found as 0.80 for the original scale and 0.86 for Turkish translation (Güleç et al., 2012, Kroenke et al., 2002).

\section{Data Analysis}

We performed descriptive analysis by calculating frequency, percentage, means, and standard deviations (SD) for presenting sociodemographic characteristics of participants. We used Pearson's chi-squared tests or Fisher's exact tests to compare categorical variables. Fisher's exact tests were preferred when at least $20 \%$ of the table had an expected count smaller than 5. Binary logistic regression was performed to identify factors related to depression, anxiety, and somatic symptoms. The cut-off score of 10 was used to determine presence of all three symptom clusters. Depression, anxiety, and somatic symptoms were separately identified as dependent variables, sociodemographic factors, and perception of threat of COVID-19 as independent variables. Statistical analyses were performed using SPSS 25.0 (Statistical Package for the Social Sciences) for Windows (SPSS, Chicago, IL). All tests conducted were two-tailed, and P value $p<0.05$ was accepted as the level of statistical significance.

\section{Results}

Sociodemographic characteristics and psychiatric symptoms of participants were illustrated in Table 1 . The study sample consisted of $315 \mathrm{HCWs}$ and the mean age was 36.35 (SD: 8.44, range from 20 to 64). Psychiatric symptoms were defined in $50.2 \%$ in our sample, $34 \%$ had depression, $23.5 \%$ had anxiety, and $37.8 \%$ had somatic symptoms (Table 1). 
Table 1.

Sociodemographic characteristics of healthcare workers

\begin{tabular}{|c|c|c|c|c|c|c|c|c|c|c|}
\hline Characteristics & $\begin{array}{c}\text { Total* } \\
(\mathrm{N}=315)\end{array}$ & $\begin{array}{c}\text { Depression } \\
(\mathrm{N}=107)\end{array}$ & $\begin{array}{c}\text { No } \\
\text { depression } \\
(\mathrm{N}=208)\end{array}$ & $p$ & $\begin{array}{l}\text { Anxiety } \\
(\mathrm{N}=74)\end{array}$ & $\begin{array}{c}\text { No } \\
\text { anxiety } \\
(\mathrm{N}= \\
241)\end{array}$ & $p$ & $\begin{array}{l}\text { Somatic } \\
\text { symptoms } \\
(\mathrm{N}=119)\end{array}$ & $\begin{array}{l}\text { No somatic } \\
\text { symptoms } \\
(\mathrm{N}=196)\end{array}$ & $p$ \\
\hline \multicolumn{11}{|l|}{ Age groups } \\
\hline$\leq 30$ years & $97(30.8)$ & $37(38.1)$ & $60(61.9)$ & \multirow{3}{*}{0.252} & $\begin{array}{c}24 \\
(23.7)\end{array}$ & $\begin{array}{c}75 \\
(76.3)\end{array}$ & \multirow{3}{*}{0.724} & $45(46.4)$ & $52(53.6)$ & \multirow{3}{*}{0.062} \\
\hline $31-40$ years & $\begin{array}{c}126 \\
(40.0)\end{array}$ & $36(28.6)$ & $90(71.4)$ & & $\begin{array}{c}27 \\
(21.4)\end{array}$ & $\begin{array}{c}99 \\
(78.6)\end{array}$ & & $39(31.0)$ & $87(69.0)$ & \\
\hline$\geq 41$ years & $92(29.2)$ & $34(37.0)$ & $58(63.0)$ & & $\begin{array}{c}24 \\
(26.1)\end{array}$ & $\begin{array}{c}68 \\
(73.9)\end{array}$ & & $35(62.0)$ & $57(38.0)$ & \\
\hline \multicolumn{11}{|l|}{ Gender } \\
\hline Male & $69(21.9)$ & $12(17.4)$ & $57(82.6)$ & \multirow{2}{*}{0.001} & $\begin{array}{c}10 \\
(14.5)\end{array}$ & $\begin{array}{c}59 \\
(85.5)\end{array}$ & \multirow{2}{*}{0.046} & $15(21.7)$ & $54(78.3)$ & \multirow{2}{*}{0.002} \\
\hline Female & $\begin{array}{c}246 \\
(78.1)\end{array}$ & 95 (38.6) & $151(61.4)$ & & $\begin{array}{c}64 \\
(26.0)\end{array}$ & $\begin{array}{c}182 \\
(74.0)\end{array}$ & & $104(42.3)$ & $142(57.7)$ & \\
\hline \multicolumn{11}{|l|}{ Marital status } \\
\hline Single & $\begin{array}{c}116 \\
(36.8)\end{array}$ & $42(36.2)$ & $74(63.8)$ & \multirow{2}{*}{0.522} & $\begin{array}{c}24 \\
(20.7)\end{array}$ & $\begin{array}{c}92 \\
(79.3)\end{array}$ & \multirow{2}{*}{0.370} & $48(41.4)$ & $68(58.6)$ & \multirow{2}{*}{0.314} \\
\hline Married & $\begin{array}{c}199 \\
(63.2)\end{array}$ & $65(32.7)$ & $134(67.3)$ & & $\begin{array}{c}50 \\
(25.1)\end{array}$ & $\begin{array}{c}149 \\
(74.9)\end{array}$ & & $71(35.7)$ & $128(64.3)$ & \\
\hline \multicolumn{11}{|l|}{ Parent status } \\
\hline No & $\begin{array}{c}122 \\
(38.7)\end{array}$ & $48(39.3)$ & $74(60.7)$ & \multirow{2}{*}{0.070} & $\begin{array}{c}31 \\
(25.4)\end{array}$ & $\begin{array}{c}91 \\
(74.6)\end{array}$ & \multirow{2}{*}{0.586} & $49(40.2)$ & $73(59.8)$ & \multirow{2}{*}{0.487} \\
\hline Yes & $\begin{array}{c}193 \\
(61.3)\end{array}$ & $59(30.6)$ & $134(69.4)$ & & $\begin{array}{c}43 \\
(22.3)\end{array}$ & $\begin{array}{c}150 \\
(77.7)\end{array}$ & & $70(36.3)$ & $123(63.7)$ & \\
\hline \multicolumn{11}{|l|}{ Monthly income } \\
\hline$\leq 5000 \mathrm{TL}$ & $\begin{array}{c}139 \\
(44.1)\end{array}$ & $54(38.8)$ & $85(61.2)$ & \multirow{3}{*}{0.031} & $\begin{array}{c}37 \\
(26.6)\end{array}$ & $\begin{array}{c}102 \\
(73.4)\end{array}$ & & $62(44.6)$ & 77 (55.4) & \multirow{3}{*}{0.001} \\
\hline 5000- $10000 \mathrm{TL}$ & $\begin{array}{c}105 \\
(33.3)\end{array}$ & $38(36.2)$ & $67(63.8)$ & & $\begin{array}{c}25 \\
(23.8)\end{array}$ & $\begin{array}{c}80 \\
(76.2)\end{array}$ & 0.290 & $44(41.9)$ & $61(58.1)$ & \\
\hline$\geq 10000 \mathrm{TL}$ & $71(22.5)$ & $15(21.1)$ & $56(78.9)$ & & $\begin{array}{c}12 \\
(16.9)\end{array}$ & $\begin{array}{c}59 \\
(83.1)\end{array}$ & & $13(18.3)$ & $58(81.7)$ & \\
\hline \multicolumn{11}{|l|}{ Cohabitant } \\
\hline Family & $\begin{array}{c}245 \\
(77.8)\end{array}$ & $81(33.1)$ & $164(66.9)$ & \multirow{2}{*}{0.525} & $\begin{array}{c}57 \\
(23.3)\end{array}$ & $\begin{array}{c}188 \\
(76.7)\end{array}$ & \multirow{2}{*}{0.859} & $84(34.3)$ & $161(65.7)$ & 0.017 \\
\hline Alone & $70(22.2)$ & $26(37.1)$ & $44(62.9)$ & & $\begin{array}{c}17 \\
(24.3)\end{array}$ & $\begin{array}{c}53 \\
(75.7)\end{array}$ & & $35(50.0)$ & $35(50.0)$ & \\
\hline Chronic diseases & & & & & & & & & & \\
\hline No & $\begin{array}{c}257 \\
(81.6)\end{array}$ & $82(31.9)$ & $175(68.1)$ & 0104 & $\begin{array}{c}59 \\
(23.0)\end{array}$ & $\begin{array}{c}198 \\
(77.0)\end{array}$ & 0637 & $84(32.7)$ & $173(67.3)$ & 0.000 \\
\hline Yes & $58(18.4)$ & $25(43.1)$ & $33(56.9)$ & 0.104 & $\begin{array}{c}15 \\
(25.9)\end{array}$ & $\begin{array}{c}43 \\
(74.1)\end{array}$ & 0.031 & $35(60.3)$ & $23(39.7)$ & 0.000 \\
\hline Smoking & & & & & & & & & & \\
\hline Yes & $98(31.1)$ & $38(38.8)$ & $60(61.2)$ & 0226 & $\begin{array}{c}24 \\
(24.5)\end{array}$ & $\begin{array}{c}74 \\
(75.5)\end{array}$ & 0779 & $42(42.9)$ & $56(57.1)$ & 0211 \\
\hline No & $\begin{array}{c}217 \\
(68.9)\end{array}$ & 69 (31.8) & $148(68.2)$ & $0.2 \angle 0$ & $\begin{array}{c}50 \\
(23.0)\end{array}$ & $\begin{array}{c}167 \\
(77.0)\end{array}$ & 0.119 & $77(35.5)$ & $140(64.5)$ & 0.211 \\
\hline Alcohol & & & & & & & & & & \\
\hline Yes & $85(27.0)$ & $38(44.7)$ & $47(55.3)$ & 0.014 & $\begin{array}{c}18 \\
(21.2)\end{array}$ & $\begin{array}{c}67 \\
(78.8)\end{array}$ & 0.556 & $37(43.5)$ & $48(56.5)$ & 0.201 \\
\hline No & $\begin{array}{c}230 \\
(73.0)\end{array}$ & $69(30.0)$ & $161(70.0)$ & 0.014 & $\begin{array}{c}56 \\
(24.3)\end{array}$ & $\begin{array}{c}174 \\
(75.7)\end{array}$ & 0.556 & $82(35.7)$ & $148(64.3)$ & 0.201 \\
\hline Physical exercise & & & & & & & & & & \\
\hline Yes & $64(20.3)$ & $14(21.9)$ & $50(78.1)$ & (0)ר & $\begin{array}{c}12 \\
(18.7)\end{array}$ & $\begin{array}{c}52 \\
(81.3)\end{array}$ & 0316 & $19(29.7)$ & $45(70.3)$ & ר \\
\hline No & $\begin{array}{c}251 \\
(79.7)\end{array}$ & $93(37.1)$ & $158(62.9)$ & 0.022 & $\begin{array}{c}62 \\
(24.7)\end{array}$ & $\begin{array}{c}189 \\
(75.3)\end{array}$ & 0.316 & $100(39.8)$ & $151(60.2)$ & 0.132 \\
\hline Occupation & & & & & & & & & & \\
\hline Nurse & $\begin{array}{c}145 \\
(46.0)\end{array}$ & $59(40.7)$ & $86(59.3)$ & & $\begin{array}{c}43 \\
(29.7)\end{array}$ & $\begin{array}{c}102 \\
(70.3)\end{array}$ & & $75(51.7)$ & $70(48.3)$ & \\
\hline Physician & $\begin{array}{c}105 \\
(33.3)\end{array}$ & $38(30.5)$ & $73(69.5)$ & 0.049 & $\begin{array}{c}23 \\
(21.9)\end{array}$ & $\begin{array}{c}82 \\
(78.1)\end{array}$ & 0.021 & $28(26.7)$ & $77(73.3)$ & 0.000 \\
\hline $\begin{array}{l}\text { Other healthcare } \\
\text { workers }\end{array}$ & $65(20.6)$ & $16(24.6)$ & $49(75.4)$ & & $8(12.3)$ & $\begin{array}{c}57 \\
(87.7)\end{array}$ & & $16(24.6)$ & $49(75.4)$ & \\
\hline Years of employment & & & & & & & & & & \\
\hline$\leq 10$ years & $\begin{array}{c}117 \\
(37.1)\end{array}$ & $45(38.5)$ & $72(61.5)$ & 0196 & $\begin{array}{c}28 \\
(23.9)\end{array}$ & $\begin{array}{c}89 \\
(76.1)\end{array}$ & & $54(46.2)$ & $63(53.8)$ & \\
\hline$\geq 10$ years & $\begin{array}{l}198 \\
(62.9)\end{array}$ & $62(31.3)$ & $136(68.7)$ & 0.196 & $\begin{array}{c}46 \\
(23.2)\end{array}$ & $\begin{array}{c}152 \\
(76.8)\end{array}$ & 0.888 & $65(32.8)$ & $133(67.2)$ & 0.018 \\
\hline Current department & & & & & & & & & & \\
\hline Isolation ward & $58(18.4)$ & $21(36.2)$ & $37(63.8)$ & & $\begin{array}{c}20 \\
(34.5)\end{array}$ & $\begin{array}{c}38 \\
(65.5)\end{array}$ & & $30(51.7)$ & $28(48.3)$ & \\
\hline Other & $\begin{array}{c}257 \\
(81.6)\end{array}$ & $86(33.5)$ & $171(66.5)$ & 0.690 & $\begin{array}{c}54 \\
(21.0) \\
\end{array}$ & $\begin{array}{c}203 \\
(79.0) \\
\end{array}$ & 0.029 & 89 (34.6) & $168(65.4)$ & 0.015 \\
\hline
\end{tabular}

*Total was not equal to the number of mental disorders because of some participants had more than one mental disorder. 
Additionally, participants' perception of threat from COVID-19 was summarized in Table 2. The results showed that $55.6 \%$ of HCWs had feel of exposure to the COVID-19, $23.5 \%$ of HCWs thought to resign, $77.8 \%$ of HCWs felt life-threatening because of COVID-19 outbreak, $57.5 \%$ of HCWs felt that family members and friends avoid to contact with them regarding their duty, and $95.6 \%$ of HCWs worried about that themselves or their family members might previously be infected (Table 2).

Table 2.

Participants' perception of threat from COVID-19

\begin{tabular}{|c|c|c|c|c|c|c|c|c|c|c|c|}
\hline \multirow[t]{2}{*}{ Characteristics } & \multicolumn{4}{|c|}{$\begin{array}{l}\text { Occupation } \\
\mathrm{N}(\%)\end{array}$} & \multicolumn{7}{|c|}{ Psychiatric Symptoms N (\%) } \\
\hline & $\begin{array}{l}\text { Total* } \\
(\mathrm{N}= \\
315)\end{array}$ & $\begin{array}{c}\text { Nurse } \\
(\mathrm{N}= \\
145)\end{array}$ & $\begin{array}{c}\text { Physician } \\
(\mathrm{N}=105)\end{array}$ & $\begin{array}{c}\text { Other } \\
\text { healthcare } \\
\text { workers } \\
(\mathrm{N}=65)\end{array}$ & $p$ & $\begin{array}{c}\text { Depression } \\
(\mathrm{N}=107)\end{array}$ & $p$ & $\begin{array}{r}\text { Anxiety } \\
(\mathrm{N}=74)\end{array}$ & $p$ & $\begin{array}{c}\text { Somatic } \\
\text { symptoms } \\
(\mathrm{N}=119)\end{array}$ & $p$ \\
\hline \multicolumn{12}{|l|}{$\begin{array}{l}\text { Do you feel } \\
\text { that you have } \\
\text { exposed to the } \\
\text { COVID-19? }\end{array}$} \\
\hline Yes & $\begin{array}{c}175 \\
(55.6)\end{array}$ & $\begin{array}{c}89 \\
(50.9)\end{array}$ & $50(28.6)$ & $36(20.6)$ & \multirow{2}{*}{0.097} & $71(40.6)$ & \multirow{2}{*}{0.006} & $\begin{array}{c}53 \\
(30.3)\end{array}$ & \multirow{2}{*}{0.001} & $81(46.3)$ & \multirow{2}{*}{0.000} \\
\hline No & $\begin{array}{c}140 \\
(44.4)\end{array}$ & $\begin{array}{c}56 \\
(40.0)\end{array}$ & $55(39.3)$ & $29(20.7)$ & & $36(25.7)$ & & $\begin{array}{c}21 \\
(15.0)\end{array}$ & & $38(27.1)$ & \\
\hline \multicolumn{12}{|l|}{$\begin{array}{l}\text { Have you ever } \\
\text { thought of } \\
\text { resigning } \\
\text { because of the } \\
\text { COVID-19 } \\
\text { outbreak? }\end{array}$} \\
\hline Yes & $\begin{array}{c}74 \\
(23.5)\end{array}$ & $\begin{array}{c}36 \\
(48.6)\end{array}$ & $24(32.4)$ & $14(18.9)$ & \multirow{2}{*}{0.858} & $43(58.1)$ & \multirow{2}{*}{0.000} & $\begin{array}{c}37 \\
(50.0)\end{array}$ & \multirow{2}{*}{0.000} & $37(50.0)$ & \multirow{2}{*}{0.013} \\
\hline No & $\begin{array}{c}241 \\
(76.5)\end{array}$ & $\begin{array}{c}109 \\
(45.2)\end{array}$ & 81 (33.6) & $51(21.2)$ & & 81 (26.6) & & $\begin{array}{c}37 \\
(15.4)\end{array}$ & & $82(34.0)$ & \\
\hline \multicolumn{12}{|l|}{$\begin{array}{l}\text { Have you } \\
\text { worried about } \\
\text { the life- } \\
\text { threatening } \\
\text { once infected? }\end{array}$} \\
\hline Yes & $\begin{array}{c}245 \\
(77.8)\end{array}$ & $\begin{array}{c}118 \\
(48.6)\end{array}$ & $80(32.7)$ & $46(18.8)$ & \multirow{2}{*}{0.170} & $88(35.9)$ & \multirow{2}{*}{0.172} & $\begin{array}{c}65 \\
(26.5)\end{array}$ & \multirow{2}{*}{0.017} & $100(40.8)$ & \multirow{2}{*}{0.037} \\
\hline No & $\begin{array}{c}70 \\
(22.2)\end{array}$ & $\begin{array}{c}26 \\
(37.1)\end{array}$ & $25(35.7)$ & $19(27.1)$ & & $19(27.1)$ & & $9(16.4)$ & & $19(27.1)$ & \\
\hline \multicolumn{12}{|l|}{$\begin{array}{l}\text { Do you feel } \\
\text { that family } \\
\text { members and } \\
\text { friends have } \\
\text { avoided } \\
\text { contact with } \\
\text { you because of } \\
\text { your work? }\end{array}$} \\
\hline Yes & $\begin{array}{c}181 \\
(57.5)\end{array}$ & $\begin{array}{c}9 \\
(49.7)\end{array}$ & $51(28.2)$ & $40(22.1)$ & \multirow{2}{*}{0.078} & $75(41.4)$ & \multirow{2}{*}{0.001} & $\begin{array}{c}50 \\
(27.6)\end{array}$ & \multirow{2}{*}{0.044} & $78(43.1)$ & \multirow{2}{*}{0.024} \\
\hline No & $\begin{array}{c}134 \\
(42.5)\end{array}$ & $\begin{array}{c}55 \\
(41.0)\end{array}$ & $54(40.3)$ & $25(18.7)$ & & 32 (23.9) & & $\begin{array}{c}24 \\
(17.9)\end{array}$ & & $41(30.6)$ & \\
\hline \multicolumn{12}{|l|}{$\begin{array}{l}\text { Worried about } \\
\text { myself or my } \\
\text { family } \\
\text { members being } \\
\text { infected by } \\
\text { COVID-19 }\end{array}$} \\
\hline Yes & $\begin{array}{c}301 \\
(95.6)\end{array}$ & $\begin{array}{c}140 \\
(46.5)\end{array}$ & 102 (33.9) & $59(19.6)$ & \multirow{2}{*}{$0.107^{* *}$} & $106(35.2)$ & \multirow{2}{*}{$0.040^{* *}$} & $\begin{array}{c}74 \\
(24.6)\end{array}$ & \multirow{2}{*}{$0.046 * *$} & $118(39.2)$ & \multirow{2}{*}{0.016} \\
\hline No & $\begin{array}{c}14 \\
(4.4)\end{array}$ & $\begin{array}{c}5 \\
(35.7)\end{array}$ & $3(21.4)$ & $6(42.9)$ & & $1(7.1)$ & & $0(0.0)$ & & $1(7.1)$ & \\
\hline
\end{tabular}

* Total was not equal to the number of mental disorders because of some participants had more than one mental disorder.

**Fisher's Exact Test

Some variables were found to be associated with presence of depression, anxiety, and somatic symptoms (Table 3). The factors associated with depression were following; females (OR, 3.85; 95\%CI, 1.71-8.69; $\mathrm{p}=0.001)$, being married (OR, 2.35; 95\%CI, 1.15-1.99; $\mathrm{P}=0.049)$, and no physical exercise (OR, 3.02; 95\% CI, 1.34-6.80; $\mathrm{p}=0.008$ ). However, only one variable was found as the risk factors for anxiety. Married HCWs were at least three times more likely to experience anxiety (OR, 3.51; 95\%CI, 1.34-9.19; $\mathrm{p}=0.010)$. As to somatic symptoms, being female (OR, 2.63 ; 95\% CI, 1.22-5.69; $\mathrm{p}=0.013)$, living alone (OR, 2.66; $95 \% \mathrm{CI}, 1.21-5.89 ; \mathrm{p}=0.016)$, having chronic diseases (OR, $4.31 ; 95 \% \mathrm{CI}, 2.12-8.74 ; \mathrm{p}=0.000)$, and no physical exercise (OR, 2.33; 95\% CI, 1.11-4.88; $\mathrm{p}=0.025)$ were found as the risk factors. Moreover, several protective factors of depression, anxiety, and somatic symptoms were 
described in Table 3. Having children (OR, 0.39; 95\%CI, $0.15-0.90 ; p=0.049$ ), monthly income level of $10.000 \mathrm{TL}$ or higher (OR, 0.12; 95\% CI, 0.02-0.40; $\mathrm{p}=0.001)$, no alcohol consumption (OR, 0.27; 95\%CI, 0.13-0.53; $\mathrm{p}=0.000$ ), not being a nurse or physician (OR, 0.37; $95 \% \mathrm{CI}, 0.16-0.85 ; \mathrm{p}=0.018$ ), not thought of resignation (OR, 0.25; 95\%CI, 0.13-0.47; $\mathrm{p}=0.000$ ), and not feel of family members and friends' avoidance (OR, 0.47; 95\%CI, .026-0.45; $\mathrm{p}=0.012$ ) were the protective factors of depression. As regards to anxiety, the following factors were protective factors; not being a nurse or physician
(OR, 0.37; 95\%CI, 0.14-0.97; $\mathrm{p}=0.043)$, not feel of exposure (OR, 0.48; 95\%CI, 0.24-0.96; $\mathrm{p}=0.037$ ), not thought of resignation $(\mathrm{OR}, 0.21 ; 95 \% \mathrm{CI}, 0.10-0.37$; $\mathrm{p}=0.000$ ). On the other hand, $10.000 \mathrm{TL}$ or higher monthly income (OR, 0.26; 95\%CI, 0.08-0.81; $\mathrm{p}=0.021)$, no alcohol consumption (OR, 0.49; 95\%CI, 0.26-0.95; $\mathrm{p}=0.034)$, not being a nurse or physician (OR, 0.26; 95\%CI, 0.12-0.56; $\mathrm{p}=0.001$ ), and not feel of exposure (OR, $0.54 ; 95 \% \mathrm{CI}, 0.30-0.98 ; \mathrm{p}=0.040$ ) were defined as the protective factors of somatic symptoms.

Table 3.

Sociodemographic characteristics and perception of threat from COVID-19 associated with depression, anxiety and somatic symptoms

\begin{tabular}{|c|c|c|c|c|c|c|c|c|c|}
\hline \multirow[t]{2}{*}{ Characteristics } & \multicolumn{3}{|c|}{ Depression } & \multicolumn{3}{|c|}{ Anxiety } & \multicolumn{3}{|c|}{ Somatic symptoms } \\
\hline & OR & $\begin{array}{c}55 \% \\
\text { CI }\end{array}$ & $\begin{array}{c}\mathrm{p}- \\
\text { value }\end{array}$ & $\mathrm{OR}$ & $\begin{array}{c}55 \% \\
\text { CI }\end{array}$ & $\begin{array}{c}\mathrm{p}- \\
\text { value }\end{array}$ & OR & $\begin{array}{c}95 \% \\
\mathrm{CI} \\
\end{array}$ & $\begin{array}{c}\mathrm{p}- \\
\text { value }\end{array}$ \\
\hline \multicolumn{10}{|l|}{ Age Groups } \\
\hline$\leq 30$ years & 1 & & & 1 & & & 1 & & \\
\hline $31-40$ years & 2.01 & $\begin{array}{c}0.60- \\
6.69\end{array}$ & 0.261 & 1.84 & $\begin{array}{c}0.49- \\
6.98\end{array}$ & 0.370 & 1.3 & $\begin{array}{l}0.40- \\
4.27\end{array}$ & 0.663 \\
\hline$\geq 41$ years & 2.04 & $\begin{array}{l}0.54- \\
7.74\end{array}$ & 0.292 & 1.87 & $\begin{array}{c}0.43- \\
8.13\end{array}$ & 0.403 & 0.99 & $\begin{array}{c}0.27- \\
3.69\end{array}$ & 0.998 \\
\hline \multicolumn{10}{|l|}{ Gender } \\
\hline Male & 1 & & & 1 & & & 1 & & \\
\hline Female & 3.85 & $\begin{array}{l}1.71- \\
8.69\end{array}$ & 0.001 & 1.78 & $\begin{array}{c}0.75- \\
4.20\end{array}$ & 0.190 & 2.63 & $\begin{array}{l}1.22- \\
5.69\end{array}$ & 0.013 \\
\hline \multicolumn{10}{|l|}{ Marital status } \\
\hline Single & 1 & & & 1 & & & 1 & & \\
\hline Married & 2.35 & $\begin{array}{l}1.15- \\
1.99\end{array}$ & 0.049 & 3.51 & $\begin{array}{l}1.34- \\
9.19\end{array}$ & 0.010 & 1.67 & $\begin{array}{l}0.74- \\
3.76\end{array}$ & 0.216 \\
\hline \multicolumn{10}{|l|}{ Parental status } \\
\hline No & 1 & & & 1 & & & 1 & & \\
\hline Yes & 0.39 & $\begin{array}{c}0.15- \\
0.90\end{array}$ & 0.049 & 0.38 & $\begin{array}{c}0.14- \\
1.03\end{array}$ & 0.057 & 1.52 & $\begin{array}{c}0.59- \\
3.89\end{array}$ & 0.387 \\
\hline \multicolumn{10}{|l|}{ Monthly income } \\
\hline$\leq 5.000 \mathrm{TL}$ & 1 & & & 1 & & & 1 & & \\
\hline $5.000-10.000 \mathrm{TL}$ & 0.52 & $\begin{array}{c}0.24- \\
1.10\end{array}$ & 0.085 & 0.68 & $\begin{array}{c}0.30- \\
1.55\end{array}$ & 0.358 & 0.89 & $\begin{array}{c}0.44- \\
1.78\end{array}$ & 0.738 \\
\hline$\geq 10.000$ & 0.12 & $\begin{array}{c}0.03- \\
0.40\end{array}$ & 0.001 & 0.29 & $\begin{array}{c}0.08- \\
1.03\end{array}$ & 0.055 & 0.26 & $\begin{array}{c}0.08- \\
0.81\end{array}$ & 0.021 \\
\hline \multicolumn{10}{|l|}{ Cohabitant } \\
\hline Family & 1 & & & 1 & & & 1 & & \\
\hline Alone & 1.25 & $\begin{array}{c}0.55- \\
2.81\end{array}$ & 0.593 & 1.37 & $\begin{array}{c}0.55- \\
3.44\end{array}$ & 0.502 & 2.66 & $\begin{array}{l}1.21- \\
5.89\end{array}$ & 0.016 \\
\hline \multicolumn{10}{|l|}{ Chronic diseases } \\
\hline No & 1 & & & 1 & & & 1 & & \\
\hline Yes & 1.94 & $\begin{array}{l}.94- \\
3.99\end{array}$ & 0.074 & 0.98 & $\begin{array}{c}0.45- \\
2.13\end{array}$ & 0.960 & 4.31 & $\begin{array}{l}2.12- \\
8.74\end{array}$ & 0.000 \\
\hline \multicolumn{10}{|l|}{ Cigar smoking } \\
\hline Yes & 1 & & & 1 & & & 1 & & \\
\hline No & 2.02 & $\begin{array}{l}0.94- \\
4.33\end{array}$ & 0.878 & 1.04 & $\begin{array}{l}0.53- \\
2.06\end{array}$ & 0.908 & 0.81 & $\begin{array}{l}0.44- \\
1.50\end{array}$ & 0.509 \\
\hline
\end{tabular}

Alcohol Consumption

Yes

No

$0.27 \quad 0.13-\quad 0.000$

0.91

$0.44-$

0.802

$\begin{array}{lcc}0.49 & 0.26- & 0.034\end{array}$ 
Physical exercise

\begin{tabular}{|c|c|c|c|c|c|c|c|c|c|}
\hline Yes & 1 & & & 1 & & & 1 & & \\
\hline No & 3.02 & $\begin{array}{l}1.34- \\
6.80\end{array}$ & 0.008 & 1.94 & $\begin{array}{c}0.84- \\
4.51\end{array}$ & 0.122 & 2.33 & $\begin{array}{l}1.11- \\
4.88\end{array}$ & 0.025 \\
\hline \multicolumn{10}{|l|}{ Occupation } \\
\hline Nurse & 1 & & & 1 & & & 1 & & \\
\hline Physician & 2.17 & $\begin{array}{l}0.81- \\
5.79\end{array}$ & 0.123 & 1.34 & $\begin{array}{l}0.49- \\
3.66\end{array}$ & 0.573 & 0.78 & $\begin{array}{c}0.32- \\
1.93\end{array}$ & 0.589 \\
\hline Other healthcare workers & 0.37 & $\begin{array}{l}0.16- \\
0.85\end{array}$ & 0.018 & 0.37 & $\begin{array}{l}0.14- \\
0.97\end{array}$ & 0.043 & 0.26 & $\begin{array}{l}0.12- \\
0.56\end{array}$ & 0.001 \\
\hline \multicolumn{10}{|l|}{ Years of employment } \\
\hline$\leq 10$ years & 1 & & & 1 & & & 1 & & \\
\hline$\geq 10$ years & 0.72 & $\begin{array}{c}0.26- \\
1.99\end{array}$ & 0.530 & 1.01 & $\begin{array}{l}0.24- \\
3.08\end{array}$ & 0.998 & 0.45 & $\begin{array}{l}0.17- \\
1.21\end{array}$ & 0.114 \\
\hline \multicolumn{10}{|l|}{ Current department } \\
\hline Isolation ward & 1 & & & 1 & & & 1 & & \\
\hline Other & 2.02 & $\begin{array}{l}0.94- \\
4.33\end{array}$ & 0.071 & 0.87 & $\begin{array}{l}0.40- \\
1.86\end{array}$ & 0.711 & 1.04 & $\begin{array}{l}0.51- \\
2.13\end{array}$ & 0.907 \\
\hline \multicolumn{10}{|l|}{$\begin{array}{l}\text { Do you feel that you have } \\
\text { exposed to the COVID-19? }\end{array}$} \\
\hline Yes & 1 & & & 1 & & & 1 & & \\
\hline No & 0.6 & $\begin{array}{c}0.33- \\
1.12\end{array}$ & 1.110 & 0.48 & $\begin{array}{l}0.24- \\
0.96\end{array}$ & 0.037 & 0.54 & $\begin{array}{c}0.30- \\
0.98\end{array}$ & 0.040 \\
\hline \multicolumn{10}{|l|}{$\begin{array}{l}\text { Have you ever thought of } \\
\text { resigning because of the } \\
\text { COVID-19 outbreak? }\end{array}$} \\
\hline Yes & 1 & & & 1 & & & 1 & & \\
\hline No & 0.25 & $\begin{array}{l}0.13- \\
0.47\end{array}$ & 0.000 & 0.21 & $\begin{array}{l}0.10- \\
0.37\end{array}$ & 0.000 & 0.58 & $\begin{array}{l}0.31- \\
1.09\end{array}$ & 0.093 \\
\hline \multicolumn{10}{|l|}{$\begin{array}{l}\text { Have you worried about the } \\
\text { life-threatening once } \\
\text { infected? }\end{array}$} \\
\hline Yes & 1 & & & 1 & & & 1 & & \\
\hline No & 0.39 & $\begin{array}{l}0.65- \\
2.97\end{array}$ & 0.390 & 0.81 & $\begin{array}{c}0.33- \\
1.98\end{array}$ & 0.635 & 1.16 & $\begin{array}{l}0.55- \\
2.42\end{array}$ & 0.698 \\
\hline \multicolumn{10}{|l|}{$\begin{array}{l}\text { Do you feel that family } \\
\text { members and friends have } \\
\text { avoided contact with you } \\
\text { because of your work? }\end{array}$} \\
\hline Yes & 1 & & & 1 & & & 1 & & \\
\hline No & 0.47 & $\begin{array}{l}0.26- \\
0.85\end{array}$ & 0.012 & 0.77 & $\begin{array}{c}0.41- \\
1.46\end{array}$ & 0.429 & 0.83 & $\begin{array}{l}0.47- \\
1.46\end{array}$ & 0.512 \\
\hline \multicolumn{10}{|l|}{$\begin{array}{l}\text { Worried about myself or my } \\
\text { family members being } \\
\text { infected by COVID-19 }\end{array}$} \\
\hline Yes & 1 & & & 1 & & & 1 & & \\
\hline No & 0.31 & $\begin{array}{l}0.03- \\
2.86\end{array}$ & 0.303 & 0.00 & $\begin{array}{c}0.00- \\
0.00\end{array}$ & 0.999 & 0.21 & $\begin{array}{c}0.23- \\
1.77\end{array}$ & 0.148 \\
\hline
\end{tabular}

\section{Discussion}

Depression, anxiety and somatic symptoms in healthcare workers during COVID-19 outbreak were investigated in this study and it was seen that those psychiatric symptoms are identified clinically significantly high in half of the workers. Among those symptoms, the highest is somatic symptoms $(37.8 \%)$ and it is followed by depression $(34.0 \%)$ and anxiety $(23.5 \%)$ respectively.

In a recent study conducted in China during COVID-19 pandemic using GAD-7 and PHQ-9, it is reported that $50.4 \%$ of the healthcare workers had depression, $44.6 \%$ had anxiety and $34 \%$ had insomnia (Lai et al., 2020). The high level of depression among HCWs might be caused by the opinion of uncertain end of the pandemic, feeling exhaustion, lack of personal protective equipment, or changed shift (Arasli et al., 2020). The reason why psychiatric symptom rates in this study were higher than our findings might be that study sample consists of healthcare workers who treat COVID-19 patients. Since working with COVID-19 patients would increase health concerns of the workers, their health concerns regarding their relatives and probably it requires more social isolation in those people, it might cause more psychiatric symptoms. In our sample, it was determined that $36.2 \%$ of the people working at isolation units had depression 
symptoms and $34.5 \%$ had anxiety symptoms. A study conducted with frontline healthcare workers in Turkey reported that $77.6 \%$ of the healthcare workers had depression, $60.4 \%$ had anxiety, $50.4 \%$ had insomnia, and $76.4 \%$ had distress symptoms (Sahin et al., 2020). Majority participants of the present study had practiced in a non-isolation ward. Therefore, the difference between the two studies' populations might cause the reason for the higher rate of psychiatric symptoms in (Sahin et al., 2020)'s study than our study. Although those rates are closer to the findings of Lai et al., the reason why they are found lower in our study is the low number of people working at isolation unit in our sample. On the other hand, in a study conducted with similar measurements among all healthcare workers in China, anxiety rate was reported as $24.1 \%$ while depression rate was $13.5 \%$ (Zhu et al., 2020). In a study conducted in Singapore, the rate of depression in healthcare workers who provide care for COVID-19 patients was reported as $10.8 \%$ (Tan et al., 2020). These results show that the COVID-19 pandemic had a direct effect on HCWs' psychological symptoms compared to previous studies before the pandemic (Cevizci and Müezzin, 2019). The reason of differences among regions might be the protective equipment differences in the hospitals, differences caused by the workers' workload, differences caused by administrative support and assurance given to the healthcare workers.

In our study, it was determined that $51.7 \%$ of healthcare workers had somatic symptoms and it was seen that this rate was higher than depression and anxiety symptoms. In the studies of Chew et al., (2020) and Barello, S., Palamenghi, L., \& Graffigna, G., (2020) somatic symptoms were also seen at the highest rate in healthcare workers during the pandemic and it was considered that it might be a way of conveying emotions (Chew et al., 2020). The relationship between somatic symptoms and emotional stress is bidirectional. The stress experienced during the pandemic might have caused somatic symptoms. In this period in which especially health anxiety is high, increased attention to somatic symptoms might have caused those somatic symptoms are graded higher.

In the risk factor analysis conducted according to sociodemographic factors, it was seen that women are at risk in terms of psychiatric symptoms, especially depression and somatic symptoms. In other studies, conducted on healthcare workers during the pandemic, it was exhibited that women are at higher risk in terms of depression (Lai et al., 2020; Sahin et al., 2020; Zhu et al., 2020,) and anxiety than men (Hacimuslar et al., 2020, Lai et al., 2020, Sahin et al., 2020; Zhu et al., 2020). That it was seen that women are at higher risk in terms of somatic symptoms in our study differs from the findings of Chew et al (2020). The reason of this difference might be related to the diversity of our study in terms of gender and cultural differences (Volkan and Volkan, 2020). Women are exposed to more stress due to the workload brought by social gender role and they might experience psychological stress (Li et al., 2020). Married individuals were found at higher risk compared to unmarried individuals in terms of both depression and anxiety. This might be caused by the fact that married individuals experience more stress with the concern of not being able to keep social distance with the family members that they live together and infecting them. It was seen that not making physical exercises is risky in terms of depression and somatic symptoms. It was seen that living alone and having a chronic disease are risky in terms of somatic symptoms. Similarly, it was reported in the study of Chew et al (2020) that those who have chronic diseases show more somatic symptoms (Chew et al., 2020). Chronic diseases might show somatic symptoms themselves or symptoms and physical restrictions caused by chronic diseases might lead to stress and psychosomatic symptoms. In addition to that, since some chronic diseases cause that COVID infection progresses heavily and therefore they might increase stress regarding infection, they might lead to development of psychosomatic symptoms.

When we look at the protective factors in terms of psychiatric symptoms among sociodemographic variables, it was seen that higher income level is protective in for both depression and anxiety. It was found that not having children is protective against depression. These findings are compatible with the study data of Zhu et al. (Zhu et al., 2020). In addition to that, it was shown in our study that absence of alcohol use which is a dysfunctional coping method is protective for depression and somatic symptoms in healthcare workers. It was seen that those who are not doctors or nurses are in the less risky group in terms of all three groups. In other studies conducted during the pandemic, it was seen that nurses and doctors are at higher risk in terms of psychiatric symptoms compared to other healthcare workers (Lai et al., 2020, Tan et al., 2020). Since nurses and doctors contact COVID-19 patients more closely and frequently and they have longer working hours compared to other healthcare workers, they might experience more stress. This might put them at a higher risk in terms of psychiatric symptoms.

According to the association of healthcare workers' COVID-19 threat perception with psychiatric symptoms, it was seen that those who think that they did not contact COVID-19 are in the less risky group in terms of anxiety and somatic symptoms. Thinking of having a sense of control over the infection has a protective function in healthcare workers against mental problems. It was shown that "more intensive training on protective equipment and infection control measures" might increase psychological resilience in healthcare workers (Ho et al., 2020, Tan et al., 2020 ,). The reason why those who think that they did not contact feel at less risk might arise from the fact that they have more sense of control over the uncertainty regarding the disease and death that the infection would cause. Those who do not consider resigning were found in the less risky group in terms of depression and anxiety. Thoughts of resigning might be the indicators of burnout which is closely related to depression, anxiety and stress level (Duarte et al., 2020). It was seen that those who answered the question 'Do you feel that family members and friends have avoided contact with you because of your work?' as "No" are at the less risky group. The reason for that might be the fact that avoiding contact is perceived as social exclusion. Seeing that relatives do not avoid contact with him/her might have reduced the healthcare worker's concerns about exclusion and stigmatization and played a protective role against depression.

One of the limitations of our study is that self-report scales were used and no clinical assessment was made. The fact that psychiatric symptoms other than depression, anxiety and somatic symptoms in the participants are not included in the assessment is another limitation. Lastly, our study 
was conducted only in Turkey and in a relatively early period of the pandemic and on a relatively limited sample, and this might limit the generalization of the results. Follow-up studies to be conducted on broader samples might help assessing the progress of psychiatric symptoms in the long term.

\section{Conclusion}

As a conclusion, our study shows that depression, anxiety and somatic symptoms progress at quite a high rate among healthcare workers. Although sociodemographic risk factors vary for depression, anxiety and somatic symptoms, it was determined that women, married individuals, those who have chronic diseases and those who do not make physical exercise are at a higher risk in terms of psychiatric symptoms. Having a higher income level, not having children, not using alcohol and not being a doctor or a nurse were identified as protective factors in terms of psychiatric symptoms. Early psychosocial and psychotherapeutic interventions to risk factor groups might be helpful in preventing the development of psychiatric symptoms.

The COVID-19 pandemic has affected the whole world, and it has mostly affected healthcare workers, in particular nurses and physicians, who have high risk to meet people having coronavirus disease. Our study found that depression, anxiety, and somatic symptoms were more prevalent among nurses and physicians compared to HCW during the pandemic. Nurses and physicians are the backbones of the health system in all countries.
Awareness of these factors might help health care planners or decision-makers to take protective measures to protect nurses and physicians. Psychological and social support systems are recommended to be developed for HCWs to strengthen their psychological health in the pandemic. Future systematic studies are needed to determine the main predictors affecting psychological health of HCWs to be aimed development of psychological support programs for HCWs and inform policymakers how healthcare systems might protect them from the effects of pandemics.

\section{Declarations}

Ethics Approval and Consent to Participate

The ethical approval of this study was obtained from the ethic committee of the University where the second author worked (decree code: 2020/54).

\section{Consent for Publication}

Not applicable.

\section{Availability of Data and Materials}

Data sets used and/or analyzed during the study can be obtained from the relevant author upon appropriate request.

\section{Competing Interests}

The authors declares that no competing interests in this manuscript.

\section{Funding}

Not applicable.

\section{Authors' Contributions}

All the authors worked all parts of the study. All authors have read and approved the final version of the article.

\section{References}

Arasli, H., Furunes, T., Jafari, K., Saydam, M. B., Degirmencioglu, Z. (2020). Hearing the voices of wingless angels: A critical content analysis of nurses' COVID-19 experiences. International Journal of Environmental Research and Public Health, 17(22), 8484.

Barello, S., Palamenghi, L., \& Graffigna, G. (2020). Burnout and somatic symptoms among frontline healthcare professionals at the peak of the Italian COVID-19 pandemic. Psychiatry Research, 290, 113129 .

Cai, H., Tu, B., Ma, J., Chen, L., Fu, L., Jiang, Y., \& Zhuang, Q. (2020). Psychological impact and coping strategies of frontline medical staff in Hunan between January and March 2020 during the outbreak of coronavirus disease 2019 (COVID- 19) in Hubei, China. Medical Science Monitor, 26, e924171-1-e924171-16.

Cevizci, O. \& Müezzin, E. (2019). Sağlık çalışanlarında psikolojik belirtilerin ve psikolojik dayanıklılığın incelenmesi [Investigation of the Psychological Symptoms and Psychological Resistance in Health Professionals]. Kibris Türk Psikiyatri ve Psikoloji Dergisi, 1(3), 166-172.

Chew, N., Lee, G., Tan, B., Jing, M., Goh, Y., Ngiam, N., ... Sharma, V. K. (2020). A multinational, multicentre study on the psychological outcomes and associated physical symptoms amongst healthcare workers during COVID-19 outbreak. Brain, Behavior, and Immunity, S0889-1591(20)30523-30527.

Duarte, I., Teixeira, A., Castro, L., Marina, S., Ribeiro, C., Jácome, C., ... \& Serrão, C. (2020). Burnout among Portuguese healthcare workers during the COVID-19 pandemic. BMC Public Health, 20(1), 1885.
Hacimusalar, Y., Kahve, A. C., Yasar, A. B., \& Aydin, M. S. (2020). Anxiety and hopelessness levels in COVID-19 pandemic: A comparative study of healthcare professionals and other community sample in Turkey. Journal of Psychiatric Research, $129,181-188$

Ho, C. S., Chee, C. Y., \& Ho, R. C. (2020). Mental Health Strategies to Combat the Psychological Impact of COVID-19 Beyond Paranoia and Panic. Annals of the Academy of Medicine, 49(1), 1.

International Council of Nurses (ICN). (2020). Retrieved July 02, 2020, from https://www.aa.com.tr/en/europe/90-000-healthcareworkers-infected-with-covid-19-icn/1831765.

Kroenke, K., Spitzer, R. L., Williams, J. B., \& Löwe, B. (2010). The Patient Health Questionnaire Somatic, Anxiety, and Depressive Symptom Scales: a systematic review. General Hospital Psychiatry, 32(4), 345-359.

Kroenke, K., Spitzer, R. L., \& Williams, J. B. (2001). The PHQ9: validity of a brief depression severity measure. Journal of General Internal Medicine, 16(9), 606-613.

Kroenke, K., Spitzer, R. L., Williams, J. B., Monahan, P. O., \& Löwe, B. (2007). Anxiety disorders in primary care: prevalence, impairment, comorbidity, and detection. Annals of Internal Medicine, 146(5), 317-325

Lai, J., Ma, S., Wang, Y., Cai, Z., Hu, J., Wei, N., ... \& Hu, S. (2020). Factors associated with mental health outcomes among health care workers exposed to coronavirus disease 2019. JAMA Network Open, 3(3), e203976. 
Li, S., Wang, Y., Xue, J., Zhao, N., \& Zhu, T. (2020). The impact of covid-19 epidemic declaration on psychological consequences: a study on active weibo users. international Journal of Environmental Research and Public Health, 17(6), 2032.

Liang, Y., Chen, M., Zheng, X., \& Liu, J. (2020). Screening for Chinese medical staff mental health by SDS and SAS during the outbreak of COVID-19. Journal of Psychosomatic Research, 133, 110102 .

Ministry of Health Turkey (MoH). (2020). Coronovirus Statistics. Retrieved July 02, 2020, from https://covid19.saglik.gov.tr/.

Ministry of Health of Turkey. (2018). Erişkin için kronik hastalıklarda fiziksel aktivite rehberi [Physical activity guide for adults with chronic diseases]. Retrieved July 02, 2020, from https://drive.google.com/file/d/1px8wZobRnrXVRSeAStSoX1w z4qYaQMgd/view

Spitzer, R. L., Kroenke, K., Williams, J. B., \& Löwe, B. (2006). A brief measure for assessing generalized anxiety disorder: The GAD-7. Archives of Internal Medicine, 166(10), 1092-1097.

Şahin, M. K., Aker, S., Şahin, G., \& Karabekiroğlu, A. (2020). Prevalence of depression, anxiety, distress and insomnia and related factors in healthcare workers during COVID-19 pandemic in Turkey. Journal of Community Health, 45(6), 1168-1177.

Tan, B., Chew, N., Lee, G., Jing, M., Goh, Y., Yeo, L., ... \& Sharma, V. K. (2020). Psychological impact of the COVID-19 pandemic on health care workers in Singapore. Annals of internal medicine, M20-1083.
Van Ravesteijn, H., Wittkampf, K., Lucassen, P., van de Lisdonk, E., van den Hoogen, H., van Weert, H., ... \& Speckens, A. (2009). Detecting somatoform disorders in primary care with the PHQ15. Annals of Family Medicine, 7(3), 232-238.

Volkan, E. \& Volkan, E. (2020). Under the COVID-19 lockdown: Rapid review about the unique case of North Cyprus. Psychological Trauma: Theory, Research, Practice, and Policy, 12(5), 539-541.

World Health Organization (WHO). (2020a. Coronavirus situation report. Retrieved July 02, 2020, from https://www.who.int/docs/default-source/coronaviruse/situationreports/20200702-covid-19-sitrep-164.pdf?sfvrsn=ac074f58_2.

World Health Organization (WHO). (2020b). Coronavirus disease (COVID-19) outbreak: Rights, roles and responsibilities of health workers, including key considerations for occupational safety and health. Retrieved July 02, 2020, from https://apps.who.int/iris/bitstream/handle/10665/331510/WHO2019-nCov-HCWadvice-2020.2-eng.pdf.

Yazici Güleç, M., Güleç, H., Simșek, G., Turhan, M., \& Aydin Sünbül, E. (2012). Psychometric properties of the Turkish version of the patient health questionnaire-somatic, anxiety, and depressive symptoms. Comprehensive Psychiatry, 53(5), 623 629.

Zhu, Z., Xu, S., Wang, H., Liu, Z., Wu, J., Li, G., ... \& Wang, W. (2020). COVID-19 in Wuhan: Sociodemographic characteristics and hospital support measures associated with the immediate psychological impact on healthcare workers. EClinical Medicine, $24,100443$. 